\title{
Estudos de Validade e Precisão do Teste de Organização de Histórias Emocionais
}

\author{
Validity and Reliability Studies of the Emotional Stories Organization Test
}

\author{
Fabiano Koich Miguel $^{1}$ e Ana Carolina Zuanazzi ${ }^{2}$
}

\begin{abstract}
Resumo
Esta pesquisa estudou o Teste de Organização de Histórias Emocionais (TOHE), um teste de desempenho em que o avaliando organiza figuras para formar uma história coerente, sendo possível escolher as reações emocionais dos personagens. Participaram 1550 pessoas brasileiras que responderam ao TOHE e, de maneira espiralada, responderam a diversas outras medidas psicológicas. O escore de Organização (i.e., organização coerente dos desenhos) mostrou-se relacionado a inteligência fluida, inteligência emocional e empatia. $\mathrm{O}$ escore de Neuroticismo (i.e., histórias com conteúdos disfóricos) relacionou-se com instabilidade emocional, baixa resistência a frustração, vivências de emoções desagradáveis e menor satisfação com a vida. O escore de Agressividade (i.e., histórias com conteúdos de agressão) relacionou-se com comportamentos agressivos, visão hostil das interações e menor sociabilidade. Além disso, o teste apresentou adequada estabilidade temporal e correlação entre seus escores. Considera-se que esses resultados constituem evidência de validade para o TOHE.
\end{abstract}

Palavras-chave: inteligência emocional, personalidade, avaliação psicológica

\begin{abstract}
This research studied the Emotional Stories Organization Test (TOHE), a performance test in which the participant organizes figures to form a coherent story, with the possibility of choosing the emotional reactions of the characters. A total of 1550 Brazilian people responded to the TOHE and to other psychological measures. The Organization score (i.e., coherent organization of the drawings) was related to fluid intelligence, emotional intelligence, and empathy. The Neuroticism score (i.e., histories with dysphoric contents) was related to emotional instability, low resistance to frustration, unpleasant experiences of emotions, and less satisfaction with life. The Aggressiveness score (i.e., stories with aggression content) was related to aggressive behaviors, hostile view of interactions, and less agreeableness. In addition, the test displayed adequate temporal stability and correlations among its scores. These results are considered validity evidence for TOHE.
\end{abstract}

Keywords: emotional intelligence, personality, psychological assessment

\footnotetext{
${ }^{1}$ Psicólogo. Doutor em Psicologia. Universidade Estadual de Londrina - CCB - Departamento de Psicologia e Psicanálise - Caixa Postal 10011 - Londrina-PR-Brasil - 86057-970. Tel. +55(43)3371-4397. E-mail: fabiano@avalpsi.com.br

${ }^{2}$ Psicóloga. Mestre em Psicologia, Doutoranda em Psicologia. Universidade São Francisco. Rua Waldemar César da Silveira, $105-$ Campinas-SP-Brasil - 13045-510. Tel.: +55(19)3779-3300. E-mail: anacarolina.zf@gmail.com
} 


\section{Introdução}

Inteligência emocional é a capacidade de utilizar o raciocínio com o objetivo de compreender as informações emocionais contidas nas experiências pessoais e sociais, e gerenciá-las para uma vivência adaptada. Tradicionalmente divide-se a inteligência emocional em quatro ramificações: a capacidade de perceber os estados emocionais em si mesmo e nas outras pessoas; a capacidade de perceber como os estados de humor influenciam o desempenho em diferentes tarefas; a capacidade de compreender por que certas situações eliciam determinadas emoções e como elas podem se alterar ao longo do tempo; e a capacidade de gerenciar os estados emocionais para promover o desenvolvimento individual e social (Mayer, Caruso, \& Salovey, 2016; Mayer, Salovey, \& Caruso, 2008).

Atualmente, é possível encontrar na literatura dois principais modelos de inteligência emocional: como capacidade cognitiva e como traço de personalidade (Roberts, MacCann, Guil, \& Mestre, 2016). O modelo cognitivo compreende a inteligência emocional como uma capacidade mental., i.e., como uma forma de raciocínio. Nesse sentido, pesquisas têm mostrado correlações com outras medidas de inteligência, enquanto as correlações com personalidade são fracas ou não significativas (MacCann, Joseph, Newman, \& Roberts, 2014; Mayer et al., 2016; Miguel, Ogaki, Inaba, \& Ribeiro, 2013; Teixeira \& Araújo, 2018). Já o modelo de traço compreende a inteligência emocional como um conjunto de percepções que o indivíduo tem sobre suas habilidades emocionais, integrando a autoavaliação das capacidades previstas no modelo cognitivo com outras características como bem-estar, autoestima, empatia, otimismo, entre outras. Pesquisas com esse modelo têm mostrado correlações maiores com traços de personalidade, enquanto as correlações com testes de inteligência são fracas ou não significativas (Ferrando et al., 2011; Petrides et al., 2016; Roberts, MacCann, Matthews, \& Zeidner, 2010; Sosa-Correa, Rodríguez-Ake, Castillo Ayuso, Ponce, \& Mestre, 2018; Woyciekoski \& Hutz, 2010).

Esses dois modelos de inteligência emocional influenciam a maneira como ela é avaliada.
Enquanto o modelo cognitivo propõe o desenvolvimento de instrumentos de desempenho, como é tradicionalmente feito com testes de inteligência, o modelo de traço utiliza inventários de autorrelato, em que o avaliando pontua em escala Likert sua percepção sobre as capacidades. Frequentemente essas duas modalidades de avaliação apresentam correlações baixas ou nulas entre si, mesmo supostamente avaliando o mesmo construto (Costa \& Faria, 2014; Di Fabio \& Saklofske, 2014; Ferguson \& Austin, 2010; Zuanazzi, Ricci, \& Miguel, 2015). Considera-se que esse fato ocorra pois capacidade e conhecimento sobre capacidade não são construtos idênticos, de maneira que os instrumentos de autorrelato exigem autoconhecimento, sendo impactados por aspectos de personalidade como autoconfiança e autoestima (Edmondson et al., 2013; Mayer et al., 2016).

Não obstante, a relação entre personalidade e raciocínio não está totalmente clara quando se trata de inteligência emocional, uma vez que mesmo o modelo cognitivo pressupõe que a regulação emocional seria influenciada por traços de personalidade individuais na escolha das estratégias de enfrentamento mais adequadas (Roberts et al., 2016). Considera-se que testes de desempenho mais integrativos, isto é, que possibilitassem a avaliação tanto de aspectos intelectuais quanto de personalidade, poderiam ser desenvolvidos. Testes cognitivos tradicionais, como raciocínio espacial ou memorização, focam em tarefas que exigem desempenho máximo, deixando pouca abertura para que aspectos de personalidade possam se manifestar (Ackerman \& Heggestad, 1997; Chamorro-Premuzic, Moutafi, \& Furnham, 2005). Já tarefas de inteligência emocional, por dizerem respeito a emoções e interações sociais, poderiam ser capazes de eliciar mais respostas influenciadas pela personalidade. De fato, Miguel e Pessotto (2016) encontraram que erros cometidos em um teste de desempenho de percepção emocional estavam associados a traços de personalidade (e.g., pessoas com níveis mais altos de agressividade tendiam a perceber mais expressões de raiva).

A presente pesquisa teve como objetivo estudar um teste desenvolvido com esse propósito, o Teste de Organização de Histórias Emocionais 
(TOHE). O TOHE é um instrumento de aplicação online que apresenta figuras embaralhadas representando uma situação e o avaliando deve organizá-las de maneira que produzam uma história com coerência cronológica. Essa modalidade de avaliação já foi utilizada nas Escalas Wechsler de Inteligência para Crianças até sua versão 3 (Wechsler, 2002), porém a criação da história era limitada à quantidade de figuras. No caso do TOHE, cada história tem uma quantidade fixa de figuras que a devem compor, porém o avaliando tem uma quantidade maior de figuras disponíveis para utilizar. Por exemplo, uma das histórias disponibiliza cinco quadros embaralhados com as seguintes figuras: uma moça chamando um ônibus; o ônibus passando direto e espirrando água na moça; a moça encharcada e brava; a moça encharcada e chorando; a moça encharcada e feliz. A história deve ser composta apenas por três quadros, permitindo que o avaliando escolha a reação emocional da personagem.

A fundamentação teórica do TOHE se aproxima do modelo de inteligência emocional compreendida como capacidade cognitiva (Mayer et al., 2008, 2016). Nesse sentido, seus itens foram construídos com base na proposta de que o avaliando deveria ser capaz de identificar o que os personagens estão sentindo, por que estão sentindo aquelas emoções e como elas transitam ao longo da história. O teste solicita também raciocínio para ordenar as figuras em uma ordem coerente. Além disso, na busca pela integração de aspectos cognitivos com aspectos de personalidade na avaliação da inteligência emocional (Roberts et al., 2016), os itens foram construídos de maneira que características de personalidade pudessem ser medidas de acordo com a maneira como o avaliando desempenha a tarefa (Ackerman \& Heggestad, 1997; ChamorroPremuzic et al., 2005; Miguel \& Pessotto, 2016). No TOHE, isso se daria de acordo com a escolha de como a história vai se desenvolver emocionalmente (e.g., se os personagens ficarão felizes, tristes, insatisfeitos, agressivos, etc.). Dessa maneira, o teste produz escores tanto de inteligência emocional (por meio da organização coerente das histórias) quanto de personalidade (por meio da escolha do tom emocional da história). Trata-se do primeiro estudo do TOHE, buscando-se estudar sua validade e precisão. Compreende-se que há carência de instrumentos de avaliação da inteligência emocional que permitam a mensuração desses dois tipos de aspectos (desempenho intelectual e traços de personalidade) e, portanto, a presente pesquisa busca preencher uma lacuna na literatura sobre inteligência emocional, uma vez que os testes já existentes avaliam exclusivamente aspectos cognitivos ou de personalidade.

Levantou-se cinco hipóteses para o funcionamento do TOHE, com base em estudos previamente realizados na área de avaliação da inteligência emocional: h1. por se tratar de desempenho cognitivo, o escore de organização estaria correlacionado com outros testes de inteligência; h2. como as histórias retratam eventos com respostas emocionais dos seus personagens, o escore de organização se correlacionaria com outras medidas de inteligência emocional; h3. a escolha das emoções dos personagens estaria correlacionada com traços de personalidade; h4. o escore de organização do TOHE (h1 e h2) se correlacionaria com os escores relacionados a personalidade (h3); h5. semelhante a outras medidas de inteligência, a precisão testereteste mostraria adequada estabilidade temporal.

\section{Método}

\section{Participantes}

Participaram da pesquisa 1550 pessoas brasileiras, com idades variando entre 18 e 40 anos $(\mathrm{M}=25,84 ; \mathrm{DP}=6,28)$, sendo $796(51,4 \%)$ do sexo feminino. As regiões do Brasil se distribuíram da seguinte maneira: 160 (10,3\%) pessoas da região Norte, $354(22,8 \%)$ da região Nordeste, $161(10,4 \%)$ da região Centro-Oeste, $616(39,7 \%)$ da região Sudeste, e 259 (16,7\%) da região Sul. A escolaridade se distribuiu da seguinte maneira: $62(4,0 \%)$ pessoas com ensino fundamental, 711 (45,9\%) com ensino médio, 661 $(42,6 \%)$ com ensino superior, e $116(7,5 \%)$ com pós-graduação. Como critério de inclusão, considerou-se pessoas entre 18 e 40 anos de idade, sem restrição de sexo, região brasileira ou escolaridade. Não foram incluídos nas análises participantes que não atendiam esses critérios. 


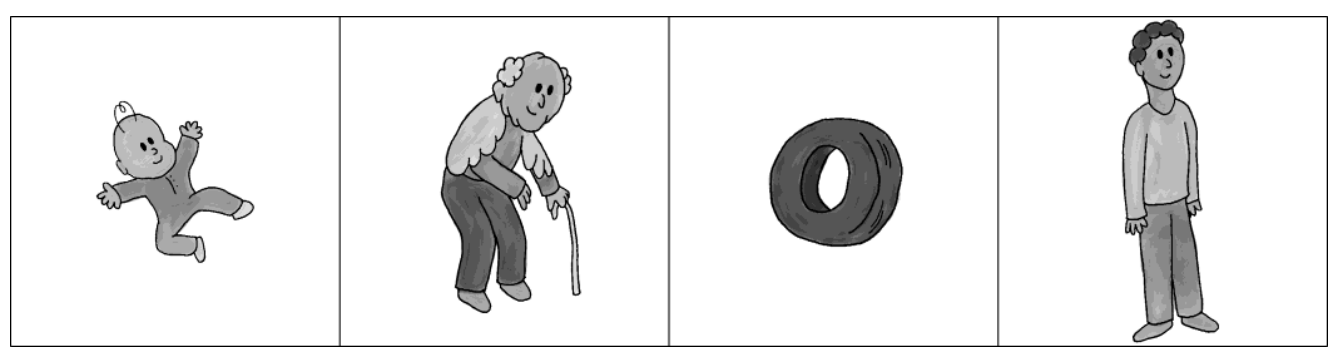

Figura 1. Itens de exemplo do Teste de Organização de Histórias Emocionais (TOHE), originalmente coloridos

\section{Instrumentos}

\section{Teste de Organização de Histórias Emocionais (TOHE)}

O TOHE foi desenvolvido para ser aplicado em plataforma online. Ele é composto por 12 histórias, incluindo o exemplo inicial. O teste apresenta no topo da tela a quantidade fixa de quadros daquela história e, abaixo, as possibilidades de figuras embaralhadas. As histórias não possuem verbalizações ou textos escritos. Todas as histórias são compostas por personagens humanos de ambos os sexos e variadas faixas etárias e etnias. Em uma história há também presença de um cachorro; em oito histórias há participação de mais de um personagem. Como apresentado, o teste foi desenvolvido para avaliar a capacidade de reconhecer os estados emocionais dos personagens e compreender como emoções surgem e transitam, o que é feito por meio da organização coerente das figuras que compõem a história. A Figura 1 mostra os desenhos do exemplo do teste, que é acompanhado das instruções. As instruções informam que a história deverá ter três quadros e ser montada em uma sequência cronológica coerente; nesse caso, primeiro o bebê, depois o adulto, e por último o idoso. O pneu, portanto, não faria parte dessa história. Nas histórias seguintes, o participante também encontraria quadros que não entrariam para a versão completa da história, devendo escolher aqueles que achasse melhor. A fim de evitar contaminação das respostas devido a vieses nas instruções, essa primeira história de exemplo não possui reações emocionais dos personagens. Um estudo piloto foi feito com quatro estudantes de graduação de Psicologia (não autores deste estudo) para se verificar a clareza das instruções e formato de aplicação. Não foram relatadas dúvidas ou alterações necessárias.
Foram criados três escores para o TOHE: Organização, Neuroticismo e Agressividade. O escore de Organização foi criado somando-se 1 ponto quando a história montada pelo participante apresentava coerência. Com o propósito de evitar que a definição do que seria uma história coerente sofresse viés da percepção dos próprios pesquisadores, utilizou-se o consenso da amostra para encontrar as produções mais frequentes. $\mathrm{O}$ consenso é utilizado em outros instrumentos de inteligência emocional por desempenho, como o Mayer-Salovey-Caruso Emotional Intelligence Test (MSCEIT), e correlaciona-se fortemente (acima de .90) com a pontuação por especialistas (Mayer, Salovey, \& Caruso, 2002). No caso do TOHE, para cada história, sequências de figuras que obtiveram frequência superior a $5 \%$ foram consideradas como coerentes e, portanto, somavam 1 ponto ao escore de Organização. Dessa maneira, na história previamente apresentada do ônibus que joga água na moça, havia três possibilidades de histórias coerentes: moça chamando ônibus; ônibus jogando água na moça; moça encharcada tendo alguma reação emocional (raiva, tristeza ou alegria). Quaisquer outras sequências (com frequência abaixo de 5\%) de fato não apresentavam coerência (por exemplo, primeiro a moça encharcada alegre; ônibus jogando água; moça encharcada brava) e receberam escore 0 .

$\mathrm{O}$ escore de Neuroticismo foi criado somando-se 1 ponto quando a história continha sentimentos disfóricos, como tristeza, medo e humilhação. O escore de Agressividade foi criado somando 1 ponto quando a história continha manifestações de raiva ou agressão. Ambos os escores foram pontuados independente da frequência da história, i.e., pela pertença daquelas emoções em alguma figura. O tempo médio de aplicação do TOHE é de 7 minutos. 


\section{Raciocínio Abstrato (RA)}

O teste RA é parte da Bateria de Provas de Raciocínio (BPR-5), composta por cinco provas de inteligência independentes (Primi \& Almeida, 2000). RA é considerado uma medida de inteligência fluida, composto por 25 itens que apresentam figuras geométricas sofrendo algum tipo de transformação (e.g., alterando tamanho, juntando-se a outra, etc.). O participante deve compreender a transformação ocorrida e aplicá-la a uma terceira figura para encontrar a alternativa correta. Para a presente amostra, o índice alfa foi .81. O tempo máximo de aplicação é de 12 minutos.

\section{Teste Informatizado de Percepção de Emoções Primárias (PEP)}

O PEP avalia a percepção emocional, uma das áreas da inteligência emocional. $O$ teste é composto por 38 vídeos breves de rostos de pessoas, sendo que os três primeiros são utilizados como exemplo. O participante deve assinalar qual ou quais emoções estão sendo expressadas em cada vídeo (Miguel \& Primi, 2014). O escore de percepção emocional é calculado por meio do modelo de Rasch (teoria de resposta ao item), e a precisão do modelo na presente amostra foi de .64. O tempo médio de aplicação é 11 minutos.

\section{Teste de Conhecimento Emocional (CE)}

O CE avalia a compreensão emocional, uma das áreas da inteligência emocional. O teste é composto por 30 histórias em formato de texto, em que diversas situações acontecem com os personagens. O participante deve assinalar quais emoções os personagens estão sentindo ou irão sentir (Oliveira \& Bueno, 2013). Para a presente amostra, o índice alfa do CE foi de .69. O tempo médio de aplicação é 10 minutos.

\section{Questionário Online de Empatia (QoE)}

O QoE é um inventário de autorrelato de 23 itens que avalia empatia, um dos componentes do traço inteligência emocional. Mais especificamente, avalia as características de compreender os sentimentos das outras pessoas, respeitar as diferenças e estar disponível para os outros (Miguel, Hashimoto, Gonçalves, Oliveira, \& Wiltenburg, 2018). Para esta pesquisa, foi utilizado o escore total de empatia, que apresentou índice alfa de .83 . O tempo médio de aplicação é 5 minutos.

\section{Escala de Dificuldades na Regulação Emocional (DERS)}

A DERS é um inventário de autorrelato (16 itens em sua versão mais breve, utilizada nesta pesquisa) que avalia déficits na regulação das emoções, estando, portanto, relacionada com a quarta área da inteligência emocional (Gratz \& Roemer, 2004). A escala avalia dificuldades em aceitar as emoções, compreender os estados emocionais, utilizar estratégias para regular o humor, controlar os impulsos e manter o foco em situações estressantes. A DERS foi traduzida e adaptada para o Brasil por Miguel, Giromini, Colombarolli, Zuanazzi e Zennaro (2017). Para esta pesquisa, foi utilizado o escore total de desregulação emocional, que apresentou índice alfa de .92. O tempo médio de aplicação é 5 minutos.

\section{Bateria Fatorial de Personalidade (BFP)}

A BFP é um inventário de autorrelato com 126 itens que avalia traços de personalidade de acordo com o modelo dos cinco grandes fatores (Nunes, Hutz, \& Nunes, 2010). Para esta pesquisa, foram utilizadas as escalas de Neuroticismo e Socialização. Neuroticismo diz respeito a desajustes emocionais, avaliando características de vulnerabilidade, instabilidade emocional, passividade e depressão. Socialização (também chamado internacionalmente de Agradabilidade) diz respeito à qualidade das interações pessoais, avaliando características de amabilidade, prósociabilidade e confiança nas pessoas. Para a presente amostra, o índice alfa foi de .92 para Neuroticismo e .85 para Socialização. O tempo médio de aplicação é 30 minutos.

\section{Inventário Dimensional Clínico da Personalidade versão 2 (IDCP-2)}

O IDCP-2 é um inventário de autorrelato de 210 itens que avaliar traços patológicos da personalidade (Carvalho \& Primi, 2018). Para esta pesquisa, foram utilizadas as escalas mais relacionadas à vivência emocional, que são Instabilidade de Humor (oscilações de humor e descontrole emocional), Evitação a Críticas (constrangimento e dificuldades de se envolver 
emocionalmente), Agressividade (comportamentos e pensamentos agressivos e impositivos) e Desconfiança (irritabilidade e crença de que os outros querem prejudicar). Para a presente amostra, os índices alfa foram .89 para Instabilidade de Humor, .89 para Evitação a Críticas, .88 para Agressividade e .89 para Desconfiança. O tempo médio de aplicação é 40 minutos.

\section{Escala de Afetos Positivos e Negativos (PANAS)}

A PANAS é um inventário de autorrelato de 20 itens que avalia a frequência da vivência de emoções positivas e negativas nos últimos dias (Watson, Clark, \& Tellegen, 1988). Apresenta dois escores: frequência de afetos positivos e negativos. A PANAS foi traduzida e adaptada para o Brasil por Giacomoni e Hutz (1997). Para a presente amostra, os índices alfa foram de .83 para positivos e .86 para negativos. O tempo médio de aplicação é 3 minutos.

\section{Escala de Satisfação de Vida (ESV)}

A ESV é um inventário de autorrelato de 5 itens que avalia o nível de contentamento geral que a pessoa sente em relação a sua vida (Diener, Emmons, Larsen, \& Griffin, 1985). A ESV foi traduzida e adaptada para o Brasil por Zanon, Bardagi, Layous e Hutz (2014). Para a presente amostra, o índice alfa foi .86. O tempo médio de aplicação é 2 minutos.

\section{Dirty Dozen (D-12)}

O D-12 é um inventário de autorrelato com 12 itens que avalia tríada sombria, i.e., aspectos negativos de personalidade como narcisismo, psicopatia e maquiavelismo (Jonason \& Webster, 2010). A escala foi traduzida e adaptada para o Brasil por Hauck Filho, Carvalho e Jonason (2015). Para a presente amostra, o índice alfa foi .87. O tempo médio de aplicação é 3 minutos.

\section{Procedimentos}

A pesquisa foi aprovada pelo Comitê de Ética em Pesquisa da Universidade Estadual de Londrina (CAAE 64469717.6.0000.5231, parecer 1.934.599). Os testes TOHE, PEP e QoE foram originalmente desenvolvidos para plataforma online, enquanto os outros testes foram adaptados para esse formato, com exceção da BFP, que foi aplicada em versão impressa. Todos os testes informatizados foram alocados em um sistema online com domínio específico.

Os convites para participação na pesquisa foram feitos por meio de redes sociais (Facebook e e-mails), com um breve texto explicando que se tratava de uma pesquisa sobre funcionamento de testes psicológicos realizada de maneira online. Junto foi disponibilizado o link de acesso para quem se interessasse. Ao clicar no link, o participante era encaminhado para o sistema online, onde o Termo de Consentimento Livre e Esclarecido (TCLE) era apresentado. Seguindo recomendações internacionais para testagem informatizada (International Test Commission, 2005), apenas o TCLE foi mostrado inicialmente, sendo que o participante concordava em participar criando um usuário e senha para acessar o sistema. Após esse cadastro, os testes eram apresentados. Dada a quantidade grande de instrumentos, a aplicação foi espiralada da seguinte maneira: o TOHE sempre era o primeiro teste disponível a ser respondido, enquanto havia rodízio para a sequência de testes seguintes. De fato, nenhum participante respondeu todos os testes, apenas $23,8 \%$ respondeu mais do que dois testes (incluindo o TOHE) e 16,1\% respondeu mais do que três testes (incluindo o TOHE). Considera-se esse resultado esperado, uma vez que a bateria completa de testes poderia levar em média 128 minutos (levando-se em conta os tempos médios de aplicação de cada teste). Por causa disso, a quantidade de participantes que respondeu os outros testes foi diferente. O Quadro 1 apresenta a distribuição de participantes de acordo com o segundo teste respondido. Buscouse manter o número próximo de 160, com distribuição semelhante de homens e mulheres. As médias de idade também foram semelhantes. Dessa maneira, além de evitar efeito de cansaço, espera-se ter reduzido efeitos de erro tipo I e II.

No caso da BFP, a aplicação foi feita com estudantes universitários de diversos cursos da Universidade Estadual de Londrina. O convite foi feito pessoalmente e os estudantes responderam o TOHE online, e a BFP na versão impressa. 
Quadro 1. Distribuição de participantes de acordo com o teste respondido além do TOHE

\begin{tabular}{lccc}
\hline Instrumento & Feminino & Masculino & Total \\
\hline RA & $102(54.8 \%)$ & $84(45.2 \%)$ & 186 \\
PEP & $83(51.9 \%)$ & $77(48.1 \%)$ & 160 \\
CE & $75(46.6 \%)$ & $86(53.4 \%)$ & 161 \\
QoE & $96(53.6 \%)$ & $83(46.4 \%)$ & 179 \\
DERS & $92(52.0 \%)$ & $85(48.0 \%)$ & 177 \\
BFP & $57(44.5 \%)$ & $71(55.5 \%)$ & 128 \\
IDCP-2 & $86(55.5 \%)$ & $69(44.5 \%)$ & 155 \\
PANAS & $101(56.7 \%)$ & $77(43.3 \%)$ & 178 \\
ESV & $101(56.7 \%)$ & $77(43.3 \%)$ & 178 \\
D-12 & $74(53.2 \%)$ & $65(46.8 \%)$ & 139 \\
\hline
\end{tabular}

\section{Análise de Dados}

Os três escores do TOHE foram correlacionados com os resultados dos outros testes e também entre si, por meio de correlação de Pearson, a fim de verificar o nível de associação entre os construtos avaliados. Os índices de correlação foram interpretados como sendo leves ao redor de .10, moderados ao redor de .30 e altos ao redor de .50 (Cohen, 1992; Vacha-Haase \& Thompson, 2004). Além disso, 40 pessoas responderam novamente ao TOHE após um período médio de 73,31 dias $(D P=53,19)$, sendo que os escores em ambos os momentos foram correlacionados entre si, a fim de verificar a estabilidade temporal.

Com base nos resultados apontados pela literatura apresentada na Introdução, as correlações foram utilizadas para se investigar as seguintes hipóteses: h1. a medida de inteligência fluida (RA) se correlacionaria positiva e moderadamente com o escore de Organização (capacidade cognitiva) e levemente com os escores de Neuroticismo e Agressividade (características de personalidade); h2. medidas de desempenho de inteligência emocional (PEP e CE) se correlacionariam positiva e moderadamente com o escore de Organização do TOHE, enquanto medidas de autorrelato de inteligência emocional (QoE e DERS) se correlacionaram positiva e moderadamente com os escores de Neuroticismo e Agressividade; h3. medidas de personalidade indicando dificuldades na vivência emocional (BFP Neuroticismo, IDCP2 Instabilidade de Humor, IDCP-2 Evitação a Críticas, PANAS e ESV) se correlacionariam positiva e moderadamente com o escore de Neuroticismo, enquanto medidas de personalidade indicando agressividade e antissociabilidade (BFP
Socialização, D-12, IDCP-2 Agressividade e IDCP-2 Desconfiança) se correlacionariam positiva e moderadamente com o escore de Agressividade; h4. os três escores do TOHE se correlacionariam significativa e moderadamente entre si; h5. como medida de precisão, o teste-reteste indicaria elevada correlação entre os escores.

Para as análises, os dados incompletos ou omissos foram removidos, considerando-se apenas os participantes que responderam aos testes de maneira completa. Para análise de testereteste, utilizou-se os escores dos participantes que espontaneamente retornaram ao sistema para responder uma segunda vez ao teste.

\section{Resultados}

As estatísticas descritivas dos instrumentos utilizados nesta pesquisa estão apresentadas no Quadro 2. Os escores do TOHE foram transformados em escores z. Portanto, os três escores apresentaram média 0,00 e desvio-padrão 1,00 .

Quadro 2. Estatísticas descritivas dos testes correlacionados com TOHE

\begin{tabular}{lcc}
\hline Escore & Média & $\begin{array}{c}\text { Desvio- } \\
\text { padrão }\end{array}$ \\
\hline RA & 18.05 & 4.36 \\
PEP Percepção & .08 & .97 \\
CE & 24.31 & 3.59 \\
QoE & -.05 & 1.13 \\
DERS & 47.31 & 15.06 \\
BFP Neuroticismo & 3.99 & 1.12 \\
BFP Socialização & 5.00 & .78 \\
IDCP-2 Agressividade & 1.93 & .60 \\
IDCP-2 Instabilidade de & 2.28 & .70 \\
Humor & 2.26 & .60 \\
IDCP-2 Desconfiança & 2.15 & .64 \\
IDCP-2 Evitação a Críticas & 29.63 & 7.68 \\
PANAS Affetos Positivos & 26.76 & 8.59 \\
PANAS Afetos Negativos & 16.62 & 7.36 \\
ESV & 26.56 & 9.65 \\
D-12
\end{tabular}

Nota. RA: Raciocínio Abstrato; PEP: Teste Informatizado de Percepção de Emoções Primárias; CE: Teste de Conhecimento Emocional; QoE: Questionário Online de Empatia; DERS: Escala de Dificuldades na Regulação Emocional; BFP: Bateria Fatorial de Personalidade; IDCP-2: Inventário Dimensional Clínico da Personalidade; PANAS: Escala de Afetos Positivos e Negativos; ESV: Escala de Satisfação de Vida; D-12: Dirty Dozen

Outros testes que utilizaram escores $\mathrm{z}$ foram PEP e QoE, e percebe-se que os participantes se mostraram dentro da expectativa normativa. Em relação aos outros testes para os quais se tem 
Quadro 3. Correlações de Pearson do TOHE com os outros instrumentos

\begin{tabular}{|c|c|c|c|}
\hline & TOHE Organização & TOHE Neuroticismo & TOHE Agressividade \\
\hline \multicolumn{4}{|l|}{ h1 } \\
\hline RA $(n=186)$ & $.27 * * *$ & .07 & -.02 \\
\hline \multicolumn{4}{|l|}{ h2 } \\
\hline PEP Percepção (n=160) & $.29 * * *$ & -.01 & -.02 \\
\hline $\mathrm{CE}(\mathrm{n}=161)$ & $.38 * * *$ & .05 & $-.17 *$ \\
\hline QoE $(n=179)$ & $.22 * *$ & $-.27 * * *$ & $-.31 * * *$ \\
\hline DERS $(n=177)$ & -.03 & $.23 * *$ & $.22 * *$ \\
\hline \multicolumn{4}{|l|}{ h3 } \\
\hline BFP Neuroticismo $(n=169)$ & $(-.03)$ & $.36^{* * *}$ & $(.23 * *)$ \\
\hline IDCP-2 Instabilidade de Humor $(n=155)$ & $(-.01)$ & $.26^{* *}$ & $(.12)$ \\
\hline IDCP-2 Evitação a Críticas $(\mathrm{n}=155)$ & $(.01)$ & $.31 * * *$ & $(.15)$ \\
\hline PANAS Afetos Positivos (n=178) & $(.09)$ & $-.36 * * *$ & $(-.23 * *)$ \\
\hline PANAS Afetos Negativos (n=178) & $(-.01)$ & $.29 * * *$ & $(.19 * *)$ \\
\hline $\operatorname{ESV}(n=178)$ & $(.07)$ & $-.35 * * *$ & $(-.26 * * *)$ \\
\hline BFP Socialização $(n=169)$ & $(.19 *)$ & $(-.17)$ & $-.32 * * *$ \\
\hline IDCP-2 Agressividade $(\mathrm{n}=155)$ & $(-.05)$ & $(.23 * *)$ & $.26 * *$ \\
\hline IDCP-2 Desconfiança $(n=155)$ & $(-.06)$ & $(.22 * *)$ & $.25^{* *}$ \\
\hline $\mathrm{D}-12(\mathrm{n}=139)$ & $(-.03)$ & $(.28 * *)$ & $.32 * * *$ \\
\hline
\end{tabular}

Nota. Os índices em parêntese indicam correlações não previstas naquela hipótese; ${ }^{*} p<.05 ; * * p<.01 ; * * * p<.001$; TOHE: Teste de Organização de Histórias Emocionais; RA: Raciocínio Abstrato; PEP: Teste Informatizado de Percepção de Emoções Primárias; CE: Teste de Conhecimento Emocional; QoE: Questionário Online de Empatia; DERS: Escala de Dificuldades na Regulação Emocional; BFP: Bateria Fatorial de Personalidade; IDCP-2: Inventário Dimensional Clínico da Personalidade; PANAS: Escala de Afetos Positivos e Negativos; ESV: Escala de Satisfação de Vida; D-12: Dirty Dozen.

conhecimento da média normativa no Brasil (i.e., RA, BFP, IDCP-2 e DERS), a amostra desta pesquisa tendeu a apresentar médias próximas das esperadas, com exceção apenas para o fator Neuroticismo da BFP, em que os participantes apresentaram médias consideravelmente mais elevadas que a normativa $(d=.80)$. Esse dado sugere uma variabilidade mais limitada de Neuroticismo, portanto as análises com esse fator foram tomadas com maior cautela.

Os três escores do TOHE foram correlacionados com os outros testes, a fim de verificar as hipóteses elencadas. Os resultados estão apresentados no Quadro 3. Os índices entre parênteses indicam análises que não estavam previstas naquela hipótese, mas que foram incluídas a título de exploração.

Em relação ao escore de Organização, todas as correlações foram significativas, variando de .22 a .33 , com exceção da correlação com a DERS, que foi próxima do nulo ( $r=-.03)$. O escore de Neuroticismo do TOHE apresentou correlações significativas com quase todos os instrumentos, variando de $r=.21$ a .36 , com exceção dos instrumento de desempenho cognitivo (RA, PEP e $\mathrm{CE}$ ). As associações mais expressivas foram com as escalas de Neuroticismo, Evitação a Críticas e afetos negativos, e negativamente com vivência de afetos positivos, satisfação de vida e qualidade de vida psicológica. $\mathrm{O}$ mesmo padrão de correlações foi encontrado para o escore de Agressividade do TOHE, que se relacionou com quase todos os testes, variando de $r=.22$ a -.32 , com exceção dos testes de desempenho cognitivo (RA e PEP) e uma correlação baixa, porém significativa, com CE.

O Quadro 4 apresenta os resultados das correlações dos escores do TOHE entre si, assim como a correlação entre as duas aplicações no teste-reteste. Os índices tenderam a ser elevados em todos os casos, com exceção da correlação entre os escores de Organização e Neuroticismo, que foi leve $(r=-.16)$.

Quadro 4. Correlações entre os escores do TOHE

\begin{tabular}{|c|c|c|c|}
\hline & 1 & 2 & 3 \\
\hline $\begin{array}{l}\text { 1. TOHE } \\
\text { Organização }\end{array}$ & $.66 * * *$ & & \\
\hline $\begin{array}{l}\text { 2. TOHE } \\
\text { Neuroticismo }\end{array}$ & $-.16 * * *$ & $.48 * * *$ & \\
\hline $\begin{array}{l}\text { 3. TOHE } \\
\text { Agressividade }\end{array}$ & $-.50 * * *$ & $.53 * * *$ & $.71 * * *$ \\
\hline
\end{tabular}

Nota. Os valores na diagonal indicam as correlações de testereteste, enquanto os valores abaixo da diagonal indicam as correlações entre os escores; ***p<.001; TOHE: Teste de Organização de Histórias Emocionais. 


\section{Discussão}

A presente pesquisa teve como objetivo estudar a validade e precisão dos escores de um instrumento novo de avaliação de aspectos da inteligência emocional, o Teste de Organização de Histórias Emocionais (TOHE). De maneira geral, entende-se que há evidências de que o TOHE avalie tanto aspectos cognitivos relacionados à compreensão das emoções quanto aspectos de personalidade relacionados ao desajustamento emocional e agressividade.

Em relação ao escore de Organização do TOHE, considerado uma representação do trabalho intelectual de organizar as figuras coerentemente de acordo com as expressões emocionais apresentadas pelos personagens, as correlações com os dois testes de desempenho de inteligência emocional foram levemente maiores do que com o teste de inteligência fluida. Esses resultados foram esperados, uma vez que o TOHE utiliza não apenas a capacidade de reconhecer uma estrutura lógica na sequência dos desenhos relacionada à inteligência fluida (h1) - mas também utiliza o reconhecimento dos estados emocionais dos personagens e compreensão das situações que os provocaram (h2). Este aspecto do teste é reforçado ao se encontrar a maior magnitude na correlação com $o$ teste de compreensão emocional, área da inteligência emocional que diz respeito a reconhecer como as emoções surgem de acordo com as situações, e podem transitar ou se alterar de acordo com os eventos subsequentes.

A magnitude da correlação com empatia foi menor do que os outros três testes de desempenho, e foi praticamente nula com desregulação emocional. Há duas interpretações possíveis para este dado. A primeira diz respeito ao formato dos instrumentos: enquanto o TOHE é de desempenho, o QoE e a DERS são de autorrelato. De fato, é possível encontrar na literatura extensa evidência de que testes que avaliam construtos semelhantes, por meio dessas duas diferentes modalidades, apresentam baixas correlações entre si (Costa \& Faria, 2014; Di Fabio \& Saklofske, 2014; Ferguson \& Austin, 2010; Zuanazzi et al., 2015). Por outro lado, os outros dois escores do TOHE (Neuroticismo e Agressividade) apresentaram correlações mais altas com inventários de autorrelato. Nesse sentido, a segunda interpretação deste resultado poderia ser que empatia e regulação emocional são construtos menos relacionados à organização de histórias emocionais do que percepção emocional, compreensão emocional e inteligência fluida. Empatia diz respeito, em parte, a compreender por que as outras pessoas estão sentindo o que estão sentindo, o que parecer ser $\mathrm{o}$ aspecto mais relacionado ao escore de Organização. Porém, empatia também engloba outros aspectos, como respeito às diferenças e disponibilidade para outras pessoas. Portanto, apenas alguns aspectos da empatia estariam relacionados ao Organização, o que explicaria a magnitude menor de correlação. Já a capacidade de regular as emoções depende mais da aquisição de um repertório de estratégias ao longo da vida. De fato, no modelo cognitivo de inteligência emocional, a regulação emocional se correlaciona pouco com as capacidade de percepção e de gerenciamento $(r=.07$ a .19$)$ (Maul, 2012; Rossen, Kranzler, \& Algina, 2008).

No que diz respeito ao escore de Neuroticismo do TOHE, a correlação maior foi com o fator Neuroticismo da BFP, o que seria esperado. Contudo, como a amostra apresentou média mais alta que a normativa nesse fator, pode ser que em outras amostras a correlação seja diferente. Não obstante, atribuir o título de Neuroticismo para esse escore do TOHE é coerente pois, no modelo dos cinco grandes fatores, neuroticismo é descrito como propensão a sofrimento psicológico, vivência de sentimentos disfóricos como ansiedade e tristeza, e baixa resistência a frustração e críticas (Nunes et al., 2010). Dessa maneira, os indivíduos mais altos em neuroticismo tenderiam a vivenciar mais sentimentos negativos e menos positivos, sentindo-se menos satisfeitos com a vida e com maior instabilidade emocional. Os resultados indicaram que essas características de personalidade estão relacionadas ao desempenho no TOHE, provavelmente fazendo com que a pessoa elabore histórias em que esses sentimentos negativos estejam presentes. Nesse ponto, o TOHE se assemelharia às técnicas projetivas, em que aspectos da personalidade que o indivíduo manifesta no mundo também se manifestam ao responder o teste (Miguel, 2014; VillemorAmaral, 2008). As correlações com os outros 
instrumentos de personalidade concordam com essa hipótese (h3), sugerindo uma tendência moderada a instabilidade do humor, assim como falta de recursos para compreender e regular as emoções, vivenciando mais emoções negativas e menor satisfação com a vida.

O mesmo pode-se dizer do escore de Agressividade (h3), que se associou principalmente a menor empatia e socialização, e a maior tríade sombria, com correlações também moderadas com comportamentos e pensamentos agressivos, e desconfiança das intenções dos outros. Todas essas características parecem apontar para uma vivência de irritabilidade e desrespeito em relação às outras pessoas, podendo o indivíduo se mostrar violento verbalmente e fisicamente em diversos contextos. Portanto, a percepção de um mundo em que as interações não são respeitosas se relacionou moderadamente com montar histórias contendo mais elementos de raiva, agressão e humilhação no TOHE.

Além disso, as correlações foram baixas entre os escores de Neuroticismo e Agressividade com os testes de inteligência. Mesmo a única correlação significativa encontrada foi em magnitude baixa ( $r=-.17)$. Esses resultados confirmam outras pesquisas relacionando personalidade com testes de desempenho, indicando que características de personalidade (no caso, Neuroticismo e Agressividade) podem influenciar no resultado de um teste de desempenho, influência essa que não é explicada por limitações intelectuais (Ackerman \& Heggestad, 1997; Chamorro-Premuzic et al., 2005; Miguel \& Pessotto, 2016).

Os três escores do TOHE apresentaram correlações significativas entre si, o que a princípio indica consistência interna (h4). Contudo, a magnitude da correlação entre Organização e Neuroticismo foi menor, indicando que essas características avaliadas pelo teste estão pouco relacionadas, ao contrário da Agressividade, que se relacionou fortemente com os dois outros escores. Uma possível explicação para esse padrão de correlações pode dizer respeito à natureza dos escores: Organização trata de desempenho cognitivo e interação dos personagens com o meio; Neuroticismo trata de aspectos de personalidade e vivência emocional interna; Agressividade trata de aspectos de personalidade e interação com o meio. Assim, percebe-se que os aspectos requeridos pelos escores de Organização e Neuroticismo se distanciam, enquanto os aspectos requeridos pelo escore de Agressividade se aproxima dos outros dois. A fim de verificar essa hipótese explicativa, estudos futuros poderiam aplicar o TOHE junto de outros testes que avaliam neuroticismo e agressividade (ou sociabilidade), conduzindo análise fatorial para verificar se há separação dessas características em dois fatores, o que indicaria maior independência dos escores do TOHE.

Outra possibilidade seria integrar os escores de Neuroticismo e Agressividade em apenas um, considerando que eles são altamente relacionados e apresentaram padrões semelhantes de correlação com os outros instrumentos. Ao se verificar as magnitudes de correlação que não foram previstas nas hipóteses iniciais (apresentadas entre parênteses no Quadro 3), percebe-se que os índices, embora levemente menores, ainda assim seguem a mesma direção. Portanto, os escores de Neuroticismo e Agressividade possivelmente apresentam um construto comum responsável pela variância compartilhada entre si e com outras medidas. Ainda, deve-se verificar a possibilidade de não apenas juntar os dois escores, mas incluir uma pontuação para as emoções positivas dos personagens. Inicialmente, a proposta dos escores de Neuroticismo e Agressividade foi criada pensando-se na exclusão mútua, i.e., quando o participante escolhesse emoções negativas, deixaria de escolher emoções positivas para os personagens. Contudo, considera-se que essa exclusão mútua nem sempre possa ser o caso: uma história poderia ser composta iniciando com emoções negativas e terminar com positivas, ou vice-versa. Dessa maneira, recomenda-se que futuros estudos desenvolvam escores de emoções positivas, relacionando-os com medidas de saúde mental e inteligência emocional, e também com os escores de emoções negativas.

Por fim, encontrou-se elevada magnitude de correlação ao se estudar o teste-reteste do TOHE (h5). Não obstante, trata-se de uma amostra ainda pequena (apenas 40 pessoas). Futuros estudos necessitariam ampliar a amostra para verificar com maior propriedade a estabilidade temporal. Trata-se de uma questão importante pois esse 
parece ser um dos poucos procedimentos possíveis para se avaliar a precisão do TOHE, uma vez que os escores do TOHE não seguem um padrão de acerto e erro como os testes de inteligência, ou escala Likert como os inventários de autorrelato, dificultando a utilização de provas estatísticas como alfa de Cronbach ou duas metades. Essa mesma limitação é encontrada nas técnicas projetivas, em que os escores são calculados por meio de classificações e não de pontuações, como no caso do TOHE. Porém, no caso dos projetivos, o cálculo da precisão costuma ser resolvido por meio da concordância entre juízes. Esse procedimento não seria possível no caso do TOHE, pois o cálculo dos escores é informatizado, não havendo necessidade de juízes.

De maneira geral, os resultados tenderam a confirmar as hipóteses h1, h2 e h3: Organização demonstrou estar moderadamente associado a percepção e compreensão emocional, inteligência fluida e alguns aspectos de empatia; Neuroticismo associou-se moderadamente a instabilidade emocional, baixa tolerância a frustração, vivências de afetos desagradáveis e menor satisfação com a vida; Agressividade associou-se moderadamente a comportamentos agressivos e impulsivos e visão hostil das interações. Considera-se que esses resultados constituem evidência de validade de construto para o TOHE, assim como há indicações de precisão e consistência interna (h4 e h5).

O formato utilizado para este teste também abre novas possibilidades para a área de avaliação da inteligência emocional, no sentido de que o TOHE é um teste de desempenho, mas que também apresenta relações moderadas com aspectos de personalidade. Dessa maneira, poderia ser encaixado tanto no modelo cognitivo quanto no modelo de traço da inteligência emocional. De fato, o escore que avalia a organização de histórias, um aspecto intelectual, apresentou magnitudes de correlação maiores com os testes de desempenho, o que é esperado no modelo cognitivo. Por outro lado, os escores que avaliam histórias disfóricas e agressivas também mostraram correlações moderadas com testes de autorrelato de personalidade, o que é esperado no modelo de traço. Um novo direcionamento para o construto inteligência emocional que integre os dois modelos já vem sendo requisitado por pesquisadores na área (Roberts et al., 2016).
Algumas limitações do estudo podem ser apontadas. Apesar da quantidade relativamente grande de pessoas que responderam ao TOHE $(\mathrm{N}=1550)$, a maioria respondeu apenas mais um teste, o que fez com que os outros testes fossem respondidos por uma quantidade bem menor (em média 160 pessoas cada). Para a maioria dos testes, a distribuição seguiu próxima à expectativa normativa, mas houve distanciamento para o fator Neuroticismo da BFP. Os participantes desta pesquisa mostraram-se com nível maior de neuroticismo, o que pode ter influenciado a magnitude das correlações por representarem, em média, uma faixa mais restrita do construto. Portanto, outras pesquisas são necessárias para verificar a estabilidade desses resultados, não apenas para o fator Neuroticismo, mas também outros testes que ainda não dispõem de normas brasileiras (PANAS e ESV).

Outra possível limitação pode ser a plataforma de aplicação do teste. A pesquisa incluiu predominantemente os participantes que tinham acesso à internet e eram cadastrados em redes sociais. Essa característica talvez não represente adequadamente a população brasileira, sendo necessário expandir os estudos com o TOHE para pessoas não incluídas digitalmente. Para isso, seria necessário que o pesquisador levasse a essas pessoas um equipamento com conexão à internet, ou as conduzisse para um laboratório.

Alguns dos instrumentos utilizados foram versões online de testes estudados originalmente em versão impressa. A literatura indica pouca diferenciação entre os desempenhos nos dois formatos (para uma revisão, ver Miguel, 2019). De fato, no caso do RA, IDCP-2 e DERS, as médias dos participantes não se afastaram das médias normativas das versões impressas. No caso do CE, PANAS, ESV e D-12, ainda não há pesquisas reportando a expectativa normativa brasileira. Não obstante, recomenda-se estudos que verifiquem a validade da versão online também dos outros instrumentos utilizados, uma vez que há acentuado aumento da informatização de instrumentos de avaliação psicológica.

Além disso, no presente estudo não se estudou a influência de outras variáveis como sexo, idade ou escolaridade. Sabe-se que essas variáveis podem estar influenciar no desempenho em algumas tarefas de inteligência emocional 
(Fernández-Berrocal, Cabello, Castillo, \& Extremera, 2012) e, por isso, recomenda-se um estudo mais aprofundado dessa questão com o TOHE.

Não obstante, o TOHE mostrou-se um instrumento promissor por integrar características cognitivas e de personalidade para avaliar aspectos da inteligência emocional. Este primeiro estudo utilizou o público geral como participantes, não especificando contextos de utilização do teste. Acredita-se que qualquer contexto em que seja necessário medir níveis de inteligência emocional (mais especificamente, a compreensão das emoções, i.e., como elas surgem, se expressam e transitam) poderia utilizar o TOHE como fonte de informação. Futuramente, contextos específicos poderiam se beneficiar de estudos mais pontuais como, por exemplo, comparações entre grupos clínicos e não-clínicos.

\section{Referências}

Ackerman, P. L., \& Heggestad, E. D. (1997). Intelligence, personality, and interests: Evidence for overlapping traits. Psychological Bulletin, 121(2), 219-245. https://doi.org/10.1037/0033-2909.121.2.219

Carvalho, L. F., \& Primi, R. (2018). Inventário Dimensional Clínico da Personalidade 2 (IDCP-2) e Inventário Dimensional Clínico da Personalidade - versão triagem (IDCPtriagem). São Paulo: Pearson.

Chamorro-Premuzic, T., Moutafi, J., \& Furnham, A. (2005). The relationship between personality traits, subjectively-assessed and fluid intelligence. Personality and Individual Differences, 38(7), 1517-1528. https://doi.org/10.1016/j.paid.2004.09.018

Cohen, J. (1992). A power primer. Psychological Bulletin, 112(1), 155-159. https://doi.org/10.1037/0033-2909.112.1.155

Costa, A. C. F., \& Faria, L. M. S. (2014). Avaliação da inteligência emocional: A relação entre medidas de desempenho e de autorrelato. Psicologia: Teoria e Pesquisa, 30(3),

339-346.

https://doi.org/10.1590/S0102-

37722014000300011

Di Fabio, A., \& Saklofske, D. H. (2014).
Comparing ability and self-report trait emotional intelligence, fluid intelligence, and personality traits in career decision. Personality and Individual Differences, 64, 174-178. https://doi.org/10.1016/j.paid.2014.02.024

Diener, E., Emmons, R. A., Larsen, R. J., \& Griffin, S. (1985). The Satisfaction with Life Scale. Journal of Personality Assessment, 49(1), 71-75. https://doi.org/10.1207/s15327752jpa4901_13

Edmondson, D., Shaffer, J. A., Chaplin, W. F., Burg, M. M., Stone, A. A., \& Schwartz, J. E. (2013). Trait anxiety and trait anger measured by ecological momentary assessment and their correspondence with traditional trait questionnaires. Journal of Research in Personality, 47(6), 843-852. https://doi.org/10.1016/j.jpp.2013.08.005

Ferguson, F. J., \& Austin, E. J. (2010). Associations of trait and ability emotional intelligence with performance on Theory of Mind tasks in an adult sample. Personality and Individual Differences, 49(5), 414-418. https://doi.org/10.1016/j.paid.2010.04.009

Fernández-Berrocal, P., Cabello, R., Castillo, R., \& Extremera, N. (2012). Gender differences in emotional intelligence: The mediating effect of age. Behavioral Psychology / Psicología Conductual: Revista Internacional Clínica y de La Salud, 20(1), 77-89.

Ferrando, M., Prieto, M. D., Almeida, L. S., Ferrándiz, C., Bermejo, R., López-Pina, J. A., ... Fernández, M. C. (2011). Trait emotional intelligence and academic performance: Controlling for the effects of IQ, personality, and self-concept. Journal of Psychoeducational Assessment, 29(2), 150159. https://doi.org/10.1177/0734282910374707

Gratz, K. L., \& Roemer, L. (2004). Multidimensional assessment of emotion regulation and dysregulation: Development, factor structure, and initial validation of the Difficulties in Emotion Regulation Scale. Journal of Psychopathology and Behavioral Assessment, 26(1), 41-54. https://doi.org/10.1023/B:JOBA.0000007455. 08539.94

Giacomoni, C. H., \& Hutz, C. S. (1997). A mensuração do bem-estar subjetivo: Escala de afeto positivo e negativo e escala de satisfação 
de vida. In Interamerican Society of Psychology (Ed.), Proceedings of the XXVI Interamerican Congress of Psychology ( $\mathrm{p}$. 313). São Paulo: IPS.

Hauck Filho, N., Carvalho, L. F., \& Jonason, P. K. (2015). Análise fatorial confirmatória da versão em português brasileiro da escala Dirty Dozen. Mesa redonda apresentada no VII Congresso Brasileiro de Avaliação Psicológica, São Paulo. Retrieved from http://www.ibapnet.org.br/congresso2015/anai s/listaresumos.htm

International Test Commission. (2005). International guidelines on computer-based and internet delivered testing. Granada, Espanha: International Test Commission.

Jonason, P. K., \& Webster, G. D. (2010). The Dirty Dozen: A concise measure of the dark triad. Psychological Assessment, 22(2), 420432. https://doi.org/10.1037/a0019265

MacCann, C., Joseph, D. L., Newman, D. A., \& Roberts, R. D. (2014). Emotional intelligence is a second-stratum factor of intelligence: Evidence from hierarchical and bifactor models. Emotion, 14(2), 358-374. https://doi.org/10.1037/a0034755

Maul, A. (2012). Higher standards of validity evidence are needed in the measurement of emotional intelligence. Emotion Review, 4(4), 411-412. https://doi.org/10.1177/1754073912446357

Mayer, J. D., Caruso, D. R., \& Salovey, P. (2016). The ability model of emotional intelligence: Principles and updates. Emotion Review, 8(4), 290-300. https://doi.org/10.1177/1754073916639667

Mayer, J. D., Salovey, P., \& Caruso, D. R. (2002). Mayer-Salovey-Caruso Emotional Intelligence Test (MSCEIT): User's manual. New York: Multi-Health Systems.

Mayer, J. D., Salovey, P., \& Caruso, D. R. (2008). Emotional intelligence: New ability or eclectic traits? American Psychologist, 63(6), 503-517. https://doi.org/10.1037/0003-066X.63.6.503

Miguel, F. K. (2014). Mitos e verdades no ensino de técnicas projetivas. Psico-USF, 19(1), 97$106 . \quad$ https://doi.org/10.1590/S141382712014000100010

Miguel, F. K. (2019). Testes informatizados. In M. N. Baptista, M. Muniz, C. T. Reppold, C.
H. S. S. Nunes, L. F. Carvalho, R. Primi, ... L. Pasquali (Eds.), Compêndio de Avaliação Psicológica (pp. 89-97). Petrópolis: Vozes.

Miguel, F. K., Giromini, L., Colombarolli, M. S., Zuanazzi, A. C., \& Zennaro, A. (2017). A Brazilian investigation of the 36- and 16-item Difficulties in Emotion Regulation Scales. Journal of Clinical Psychology, 73(9), 11461159. https://doi.org/10.1002/jclp.22404

Miguel, F. K., Hashimoto, E. S., Gonçalves, E. R. D. S., Oliveira, G. T., \& Wiltenburg, T. D. (2018, no prelo). Estudos de validade do Questionário Online de Empatia. Temas Em Psicologia, 26(4).

Miguel, F. K., Ogaki, H. A., Inaba, C. M., \& Ribeiro, D. O. (2013). Percepção emocional e inteligência: Contribuições para o modelo CHC. Revista Sul-Americana de Psicologia, 1(1), 36-47.

Miguel, F. K., \& Pessotto, F. (2016). Projective aspects on cognitive performance: Distortions in emotional perception correlate with personality. Psicologia: Reflexão e Crítica, 29(1), 17. https://doi.org/10.1186/s41155-0160036-6

Miguel, F. K., \& Primi, R. (2014). Estudo psicométrico do Teste Informatizado de Percepção de Emoções Primárias. Avaliação Psicológica, 13(1), 1-9.

Nunes, C. H. S. S., Hutz, C. S., \& Nunes, M. F. O. (2010). Bateria Fatorial de Personalidade (BFP): Manual técnico. São Paulo: Casa do Psicólogo.

Oliveira, A. E. N. A., \& Bueno, J. M. H. (2013). Construção e avaliação das propriedades psicométricas de um instrumento para avaliação do conhecimento emocional. Pôster apresentado no VI Congresso Brasileiro de Avaliação Psicológica, Maceió. Retrieved from

http://www.ibapnet.org.br/congresso2013/lista _trabalhos_poster.php

Petrides, K. V., Mikolajczak, M., Mavroveli, S., Sanchez-Ruiz, M.-J., Furnham, A., \& PérezGonzález, J.-C. (2016). Developments in trait emotional intelligence research. Emotion Review, 8(4), 335-341. https://doi.org/10.1177/1754073916650493

Primi, R., \& Almeida, L. S. (2000). Bateria de Provas de Raciocínio (BPR-5): Manual 
técnico. São Paulo: Casa do Psicólogo.

Roberts, R. D., MacCann, C., Guil, R., \& Mestre, J. M. (2016). Reimagining emotional intelligence: A healthy, much needed, and important progression for the field. Emotion Review, 8(4), 334-334. https://doi.org/10.1177/1754073916650506

Roberts, R. D., MacCann, C., Matthews, G., \& Zeidner, M. (2010). Emotional intelligence: Toward a consensus of models and measures. Social and Personality Psychology Compass, $4(10)$, 821-840. https://doi.org/10.1111/j.17519004.2010.00277.x

Rossen, E., Kranzler, J. H., \& Algina, J. (2008). Confirmatory factor analysis of the MayerSalovey-Caruso Emotional Intelligence Test v2.0 (MSCEIT). Personality and Individual Differences, 44(5), 1258-1269. https://doi.org/10.1016/j.paid.2007.11.020

Sosa-Correa, M., Rodríguez-Ake, A., Castillo Ayuso, R., Ponce, N., \& Mestre, J. (2018). Propiedades psicométricas: Escala Yucatán del Uso Percibido de la Inteligencia Emocional en Menores (EYUPIE-M). Revista Iberoamericana de Diagnóstico y Evaluación - e Avaliação Psicológica, 48(3), 127-138. https://doi.org/10.21865/RIDEP48.3.11

Teixeira, F., \& Araújo, A. (2018). Propriedades psicométricas do Mayer-Salovey-Caruso Emotional Intelligence Test - MSCEIT V2.0: Uma revisão sistemática da ;iteratura. Revista Iberoamericana de Diagnóstico y Evaluación - e Avaliação Psicológica, 48(3). https://doi.org/10.21865/RIDEP48.3.14

Vacha-Haase, T., \& Thompson, B. (2004). How to estimate and interpret various effect sizes. Journal of Counseling Psychology, 51(4), 473-481. https://doi.org/10.1037/00220167.51.4.473

Villemor-Amaral, A. E. (2008). A validade teórica em avaliação psicológica. Psicologia: Ciência e Profissão, 28(1), $\quad$ 98-109. https://doi.org/10.1590/S141498932008000100008

Watson, D., Clark, L. A., \& Tellegen, A. (1988). Development and validation of brief measures of positive and negative affect: The PANAS scales. Journal of Personality and Social Psychology, 54(6), 1063-1070. https://doi.org/10.1037/0022-3514.54.6.1063

Wechsler, D. (2002). WISC III - Escala Wechsler de inteligência para crianças: Manual. São Paulo: Casa do Psicólogo.

Woyciekoski, C., \& Hutz, C. S. (2010). Inteligência emocional avaliada por autorrelato difere do construto personalidade? Psico-USF, 15(2), 151-159. https://doi.org/10.1590/S141382712010000200003

Zanon, C., Bardagi, M. P., Layous, K., \& Hutz, C. S. (2014). Validation of the Satisfaction with Life Scale to Brazilians: Evidences of measurement noninvariance across Brazil and US. Social Indicators Research, 119(1), 443453. https://doi.org/10.1007/s11205-013-04785

Zuanazzi, A. C., Ricci, D. S., \& Miguel, F. K. (2015). Avaliação da alexitimia e percepção emocional: Comparação entre autorrelato e desempenho. Temas em Psicologia, 23(4), 831-842. https://doi.org/10.9788/TP2015.4-03 